\title{
Space-time correlation models and contaminant plumes
}

\author{
D. E. Myers*, $\dagger$ \\ Department of Mathematics, University of Arizona, Tucson, AZ 85721, U.S.A.
}

\begin{abstract}
SUMMARY
A contaminant plume might be described by a function defined in space-time. Spatial integrals or time derivatives of this function as well as time derivatives of spatial integrals will quantify characteristics such as the total volume of the plume, the total concentration of the contaminant in the plume, rates of change of the volume, and rates of change of concentration.

The plume function usually cannot be derived in analytic form but instead must be estimated or approximated. The dual form of the kriging estimator, which is equivalent to the use of radial basis functions, provides a tool for modeling this function in analytic form. The extension of the kriging estimator, in its usual form or in its dual form, to space-time poses no problems since the estimator and the equations are essentially dimension free. The difficulty is an adequate choice of space-time variograms or covariances.

The product-sum and integrated product-sum models provide an extensive array of valid models and also lead to a simple process for fitting the models by the use of marginal variograms. Examples are given and an application to air pollution data from the Milan District (Italy) illustrates the method. Copyright (C) 2002 John Wiley \& Sons, Ltd.
\end{abstract}

KEY WORDS: dual kriging; space-time variograms; product-sum models; marginal variograms; radial basis functions

\section{INTRODUCTION}

An air pollution plume or a contaminant plume in the subsurface cannot easily be directly measured or characterized as an object. In the case of a visible air pollution plume, it might be recorded on film or video. This mode does not lend itself to analysis of the plume characteristics such as shape, total volume, rate of change of volume, rate of movement or spreading. Most often the plume will be observed by taking measurements, i.e. samples at a small number of observation sites. At each site, samples will be collected or recorded at regular time intervals although there may be some missing time points for some locations. Each location-time can be viewed as a point in $R^{k} \times T, R^{k}$ being $k$ dimensional Euclidean space and $T$ representing time. Let the data points in space-time be denoted by $\left(\mathbf{s}_{i}, t_{i}\right), i=1, \ldots, n$. Denote the contaminant concentration at $(\mathbf{s}, t)$ by $Z(\mathbf{s}, t)$. If $Z(\mathbf{s}, t)$ were known in analytical form then most of the characteristics of the plume could be determined from this function.

*Correspondence to: D. E. Myers, Department of Mathematics, University of Arizona, Tucson, AZ 85721, U.S.A.

${ }^{\dagger}$ E-mail: myers@math.arizona.edu

Copyright (C) 2002 John Wiley \& Sons, Ltd. 
In a later section we will discuss the plume characteristics that can be described in terms of $Z(\mathbf{s}, t)$. Since of course $Z(\mathbf{s}, t)$ is not known it must be estimated or approximated from data. Then the plume characteristics will be estimated by using the approximating function in lieu of the unknown $Z(\mathbf{s}, t)$. The dual form of kriging is a logical choice for generating the approximating function; this is essentially equivalent to using radial basis functions (Myers, 1988, 1991, 1992). However to use kriging, one must have a valid space-time variogram or covariance. Although $R^{k} \times T$ might be thought of as simply a $k+1$ dimensional space and begin with an isotropic model with a suitable anisotropy applied at the time of kriging, this approach ignores the fundamental difference between time and distance in space.

Kriging has been widely used for the analysis of air pollution and to a lesser extent ground water contamination studies. However, these studies have mostly been of the 'static' nature, i.e. one considers only a snapshot in time. The principal difficulty has been the lack of a family of valid spacetime covariances or variograms. Kyriakidis and Journel (1999), Posa (1993), Rodriguez-Iturbe and Meija (1974) and Rouhani and Hall (1989) review the problems associated with space-time modeling. In addition, the usual approach in geostatistics is to simply interpolate values at non-data locations rather than to generate an interpolating function although such a function is implicit in the use of the kriging estimator. One of the advantages of using the interpolating function is that it provides a tool for estimating non-directly observable characteristics of a contaminant plume.

There are two main parts in this paper: (i) showing how to construct a much larger class of valid space-time variograms and how to use data to fit them; (ii) the use of dual kriging to estimate and approximate various plume characteristics. The principal focus will be on (i).

\section{PLUME CHARACTERISTICS}

It is necessary to first state how the function $Z(\mathbf{s}, t)$ is assumed to relate to the plume. Since the values of this function represent the concentration(s) of the contaminant(s) at the space-time point $(\mathbf{s}, t)$, the 'plume' is the set of points where $Z(\mathbf{s}, t)>0$. More precisely let

$$
\mathbf{P}(t)=\{\mathbf{s} \mid Z(\mathbf{s}, t)>0\} .
$$

$\mathbf{P}(t)$ is then a set of points in space and can change with time. Properties of the plume will first be described in terms of the (unknown) function $Z(\mathbf{s}, t)$, then later it will be shown that these can be approximated or estimated by the use of dual kriging. Let $z$ be a real number (the definition would have to be modified slightly for vector valued functions),

$$
I_{Z}(\mathbf{s}, t ; z)=1 \quad \text { if } Z(\mathbf{s}, t) \leq z \text { and zero otherwise. }
$$

This is the usual way of defining the indicator transform in geostatistics. Then the volume of the plume at time $t$ is given by

$$
\mathbf{V}(t)=\int_{R^{k}}\left[1-I_{Z}(\mathbf{s}, t ; 0)\right] \mathrm{d} \mathbf{s} .
$$

If the plume is bounded in space then the boundedness of the integrand is sufficient to ensure that the integral exists. Because instrumentation and/or analytical procedures will nearly always have a 
detection limit that is greater than zero, when using data to estimate the plume volume it will be more appropriate to use a slightly positive cut-off value in lieu of zero. The rate of change of the volume of the plume would then be given by the time derivative,

$$
\frac{\mathrm{d}}{\mathrm{d} t} \mathbf{V}(t)
$$

While it might not be reasonable to expect the indicator function to be smooth as a function of the space coordinates, it is more reasonable to expect it to be smooth, i.e. differentiable with respect to the time variable.

The total amount of contaminant in the plume at time $t$ is given by

$$
\mathbf{T}(t)=\int_{\mathbf{P}(t)} Z(\mathbf{s}, t) \mathrm{d} \mathbf{s} .
$$

The total contaminant concentration in the plume may be of interest because in some instances it will be possible to determine the total pollution independently of estimating this integral and as such would allow a check on the modeling of the plume function. Of course the average concentration of the contaminant in the plume would be given by

$$
\mathbf{T}(t) / \mathbf{V}(t)
$$

and the rate of change of the average concentration is given by

$$
\frac{\mathrm{d}}{\mathrm{d} t} \mathbf{T}(t) / \mathbf{V}(t)
$$

In some instances it might be useful to compute the average of a fixed spatial area rather than over the entire plume. Of course the rate of change of the total concentration would be given by

$$
\frac{\mathrm{d}}{\mathrm{d} t} \mathbf{T}(t)
$$

If the function $Z(\mathbf{s}, t)$ is sufficiently smooth with respect to time, then

$$
\mathbf{R}(\mathbf{s})=\frac{\mathrm{d}}{\mathrm{d} t} Z(\mathbf{s}, t)
$$

would be the local rate of change of concentration. The set of points, in space, where this derivative is zero would represent a stagnant area.

\section{SOME PRELIMINARIES}

Since it is proposed to approximate the plume function $Z(\mathbf{s}, t)$ by dual (space-time) kriging we review some basics and fix the notation. The usual kriging formulation requires the use of a random function 
model for $Z(\mathbf{s}, t)$, but the dual kriging formulation does not explicitly require this, and Madych and Nelson (1988) have given such a construction. Although in the radial basis function literature there are no examples of the use of anisotropies, the theory does not exclude it. For simplicity and for consistency with the geostatistical literature, the language of random functions is used. The basis functions (variogram, covariance) are described in that context. Assume that $Z(\mathbf{s}, t)$ is either second order stationary or intrinsic stationary. Recall that the random function is second order stationary if:

$$
\begin{gathered}
\mathrm{E}[Z(\mathbf{s}, t)]=\text { constant } \\
C_{s t}\left(\mathbf{h}_{s}, h_{t}\right)=\operatorname{Cov}\left[Z\left(\mathbf{s}+\mathbf{h}_{s}, t+h_{t}\right), Z(\mathbf{s}, t)\right] .
\end{gathered}
$$

Both exist for any $\mathbf{s}, \mathbf{h}_{s}, t, h_{t}$ and $C_{s t}\left(\mathbf{h}_{s}, h_{t}\right)$ is only a function of $\mathbf{h}_{s}, h_{t}$ where $\mathbf{h}_{s}$ is an increment vector in space and $h_{t}$ is an increment in time. The covariance function must be positive definite (not just semi-definite).

The random function is intrinsic stationary if the variogram

$$
\mathrm{E}\left[Z\left(\mathbf{s}+\mathbf{h}_{s}, t+h_{t}\right)-Z(\mathbf{s}, t)\right]=0
$$

and

$$
\gamma_{s t}\left(\mathbf{h}_{s}, h_{t}\right)=0.5 \operatorname{Var}\left[Z\left(\mathbf{s}+\mathbf{h}_{s}, t+h_{t}\right)-Z(\mathbf{s}, t)\right]
$$

exist for any $\mathbf{s}, \mathbf{h}_{s}, t, h_{t}$ and $\gamma_{s t}\left(\mathbf{h}_{s}, h_{t}\right)$ is only a function of $\mathbf{h}_{s}, h_{t}$, where again $\mathbf{h}_{s}$ is an increment vector in space and $h_{t}$ is an increment in time. The variogram must be conditionally negative definite (not semi-definite). Positive definiteness is sufficient to ensure that a covariance is bounded; conditional negative definiteness is not sufficient for boundedness and a separate growth condition must be imposed, namely less than quadratic.

The extension of the covariance function or variogram to space-time might be thought of as simply an extension to a higher order dimensional space. In that case one could generate models in the higher dimensional space in the usual way, i.e. one begins with an isotropic (spatial) covariance $C(r)$ or variogram $\gamma(r)$. Define a distance function $\left|\left(\mathbf{h}_{s}, h_{t}\right)\right|$, then $C_{s t}\left(\mathbf{h}_{s}, h_{t}\right)=C\left(\left|\mathbf{h}_{s}, h_{t}\right|\right)$ and $\gamma_{s t}\left(\mathbf{h}_{s}, h_{t}\right)=$ $\gamma\left(\left|\mathbf{h}_{s}, h_{t}\right|\right)$ are valid space-time models. The difficulty is in choosing or constructing the distance function. An obvious choice might be $\left[\left|\mathbf{h}_{s}\right|^{2}+c^{2}\left|h_{t}\right|^{2}\right]^{0} 0.5$ for some choice of the constant $c^{2}$. $\left|\mathbf{h}_{s}\right|$ is the usual Euclidean metric and $\left|h_{t}\right|$ is absolute value. This construction has been used but it ignores the fundamental difference between time and a Euclidean dimension. Although metric models are not the focus of this discussion, it will be found that some models constructed in another fashion appear as metric models.

\section{A SIMPLE CONSTRUCTION FOR SPACE-TIME MODELS}

An alternative to the use of a metric is to use a construction analogous to a zonal anisotropy, i.e. to use models that separately depend only on space and only on time and then combine them in an appropriate fashion. It is well known (Myers and Journel, 1990; Rouhani and Myers, 1990) that the 
sum of two variograms or the sum of two covariances is generally not valid. That is, if $C_{s}\left(\mathbf{h}_{s}\right)$ is a valid covariance in space and $C_{t}\left(h_{t}\right)$ is a valid covariance in time, the sum

$$
C_{s}\left(\mathbf{h}_{s}\right)+C_{t}\left(h_{t}\right)
$$

is in general only semi-definite and can lead to non-invertible kriging matrices. The same is true for the sum of a space variogram and a time variogram. Although the product of a space variogram and a time variogram would generally not satisfy the growth limitation, the product of a space covariance and a time covariance will lead to a valid model. This model is somewhat limited but it provides the motivation for a more general construction; it is also useful to consider the variogram model determined by the product covariance model.

\section{THE PRODUCT MODEL}

Let $C_{s}\left(\mathbf{h}_{s}\right), C_{t}\left(h_{t}\right)$ be valid spatial and temporal covariances; then

$$
C_{s t}\left(\mathbf{h}_{s}, h_{t}\right)=C_{s}\left(\mathbf{h}_{s}\right) \cdot C_{t}\left(h_{t}\right)
$$

is a valid space-time covariance. Let $\gamma_{s t}\left(\mathbf{h}_{s}, h_{t}\right), \gamma_{s}\left(\mathbf{h}_{s}\right), \gamma_{t}\left(h_{t}\right)$ be the corresponding variograms, i.e.

$$
\begin{gathered}
\gamma_{s t}\left(\mathbf{h}_{s}, h_{t}\right)=C_{s t}(0,0)-C_{s t}\left(\mathbf{h}_{s}, h_{t}\right) \\
\gamma_{s}\left(\mathbf{h}_{s}\right)=C_{s}(0)-C_{s}\left(\mathbf{h}_{s}\right)
\end{gathered}
$$

and

$$
\gamma_{t}\left(h_{t}\right)=C_{t}(0)-C_{t}\left(h_{t}\right)
$$

Then

$$
\gamma_{s t}\left(\mathbf{h}_{s}, h_{t}\right)=C_{t}(0) \cdot \gamma_{s}\left(\mathbf{h}_{s}\right)+C_{s}(0) \cdot \gamma_{t}\left(h_{t}\right)-\gamma_{s}\left(\mathbf{h}_{s}\right) \cdot \gamma_{t}\left(h_{t}\right) .
$$

The model in this form has several interesting and useful properties:

$$
\gamma_{s t}\left(\mathbf{h}_{s}, 0\right)=C_{t}(0) \cdot \gamma_{s}\left(\mathbf{h}_{s}\right)
$$

and

$$
\gamma_{s t}\left(0, h_{t}\right)=C_{s}(0) \cdot \gamma_{t}\left(h_{t}\right)
$$

Thus estimating and modeling $\gamma_{s t}\left(0, h_{t}\right)$ is nearly equivalent to estimating and modeling $\gamma_{t}\left(h_{t}\right)$. Likewise estimating and modeling $\gamma_{s t}\left(\mathbf{h}_{s}, 0\right)$ is nearly equivalent to estimating and modeling $\gamma_{s}\left(\mathbf{h}_{s}\right)$. The sills $C_{t}(0), C_{s}(0)$ must also be determined. 
The usual sample variograms can be modified for estimating and modeling $\gamma_{s t}\left(0, h_{t}\right)$ and $\gamma_{s t}\left(\mathbf{h}_{s}, 0\right)$ :

$$
\begin{aligned}
& \gamma_{s t}^{*}\left(0, h_{t}\right)=\frac{1}{N(\mathbf{s})} \sum_{s} \frac{1}{N_{\mathbf{s}}\left(h_{t}\right)} \sum_{h_{t}}\left[Z\left(\mathbf{s}, t+h_{t}\right)-Z(\mathbf{s}, t)\right]^{2} \\
& \gamma_{s t}^{*}\left(\mathbf{h}_{s}, 0\right)=\frac{1}{N(t)} \sum_{t} \frac{1}{N_{t}\left(\mathbf{h}_{s}\right)} \sum_{h_{s}}\left[Z\left(\mathbf{s}+\mathbf{h}_{s}, t\right)-Z(\mathbf{s}, t)\right]^{2} .
\end{aligned}
$$

\section{Case Study 1}

Monthly average $\mathrm{SO}_{2}$ data collected at 33 locations in the Lombardy region of Italy from January 1983 to December 1986 were analyzed by De Cesare et al., 1997. The data were first deseasonalized in time by fitting to a time series using moving averages. A space-time variogram was fitted using the residuals. A product model was used with components

$$
\gamma_{s}\left(\mathbf{h}_{s}\right)=270 \delta\left(\mathbf{h}_{s}\right)+1884 \operatorname{Sph}\left(\mathbf{h}_{s} / 4000\right)
$$

and

$$
\gamma_{t}\left(h_{t}\right)=154 \delta\left(h_{t}\right)+\operatorname{Sph}\left(h_{t} / 4.8\right)
$$

The variogram fit was evaluated in two ways: the usual cross-validation using the jackknife and then predicting values into 1987 using only the earlier data. The 1987 data was not used in the variogram modeling process nor as data for prediction, hence statistics such as the mean square normalized error could be used to evaluate the variogram fit. Further details are found in De Cesare (1997).

\section{PRODUCT-SUM MODELS}

Although the sum of a spatial and a temporal variogram is not a valid space-time variogram, adding a spatial variogram or temporal variogram to a valid space-time model can result in a valid model as is seen in the variogram form of the product model. This suggests the use of a product-sum model. It will be given first in covariance form and then re-written in variogram form. Let

$$
C_{s t}\left(\mathbf{h}_{s}, h_{t}\right)=k_{1} C_{s}\left(\mathbf{h}_{s}\right) \cdot \mathbf{C}_{t}\left(h_{t}\right)+k_{2} C_{s}\left(\mathbf{h}_{s}\right)+k_{3} C_{t}\left(h_{t}\right)
$$

where $C_{t}$ and $C_{s}$ are valid temporal and spatial covariance models, respectively. For positive definiteness it is then sufficient that $k_{1}>0, k_{2} \geq 0$ and $k_{3} \geq 0$. In terms of the variograms the model becomes

$$
\gamma_{s t}\left(\mathbf{h}_{s}, h_{t}\right)=\left(k_{2}+k_{1} C_{t}(0)\right) \gamma_{s}\left(\mathbf{h}_{s}\right)+\left(k_{3}+k_{1} C_{S}(0)\right) \gamma_{t}\left(h_{t}\right)-k_{1} \gamma_{s}\left(\mathbf{h}_{s}\right) \cdot \gamma_{t}\left(h_{t}\right)
$$


where $\gamma_{s}$ and $\gamma_{t}$ are the coresponding spatial and temporal variogram models with sills $C_{s}(0)$ and $C_{t}(0)$. The second-order stationarity assumption is sufficient to ensure that these variograms have sills. The conditions analogous to Equations (17) and (18) are

$$
\gamma_{s t}\left(\mathbf{h}_{s}, 0\right)=\left(k_{2}+k_{1} C_{t}(0)\right) \gamma_{s}\left(\mathbf{h}_{s}\right)
$$

and

$$
\gamma_{s t}\left(0, h_{t}\right)=\left(k_{3}+k_{1} C_{s}(0)\right) \gamma_{t}\left(h_{t}\right)
$$

As in the case of the product model, estimating and modeling $\gamma_{s t}\left(0, h_{t}\right)$ is nearly equivalent to estimating and modeling $\gamma_{t}\left(h_{t}\right)$. Likewise, estimating and modeling $\gamma_{s t}\left(\mathbf{h}_{s}, 0\right)$ is nearly equivalent to estimating and modeling $\gamma_{s}\left(\mathbf{h}_{s}, 0\right)$. These two relationships can be further simplified by imposing three constraints:

$$
\begin{aligned}
k_{2}+k_{1} C_{t}(0) & =1 \\
k_{3}+k_{1} C_{s}(0) & =1 \\
k_{3}+k_{2}+k_{1} & =1 .
\end{aligned}
$$

\section{Case Study 2}

De Cesare et al. (2000a, 2000b) consider $\mathrm{NO}_{2}$ data from the Lombardy region, Italy. There were 48 spatial locations, hourly average measurements for a period of one year. The data were first deseasonalized by fitting to a time series using moving averages. The following models were used:

$$
\begin{aligned}
\gamma_{s}\left(\mathbf{h}_{s}\right) & =220 \operatorname{Sph}\left(\left|\mathbf{h}_{s}\right| / 2000\right)+450\left[1-\exp \left(-\left|\mathbf{h}_{s}\right| / 18000\right)\right] \\
\gamma_{t}\left(h_{t}\right) & =280 \operatorname{Sph}\left(\left|h_{t}\right| / 12\right)+90 \operatorname{Sph}\left(\left|h_{t}\right| / 24\right)+250 \operatorname{Sph}\left(\left|h_{t}\right| / 96\right) .
\end{aligned}
$$

Details are found in De Cesare et al. (2000a).

\subsection{The unconstrained product-sum model}

Simply write

$$
\begin{aligned}
& k_{2}+k_{1} C_{t}(0)=k_{s} \\
& k_{3}+k_{1} C_{s}(0)=k_{t} .
\end{aligned}
$$

Then

$$
\gamma_{s t}\left(\mathbf{h}_{s}, 0\right)=k_{s} \gamma_{s}\left(\mathbf{h}_{s}\right)
$$

and

$$
\gamma_{s t}\left(0, h_{t}\right)=k_{t} \gamma_{t}\left(h_{t}\right)
$$


Thus $k_{s}$ and $k_{t}$ can be viewed as coefficients of proportionality between the space-time variograms $\gamma\left(\mathbf{h}_{s}, 0\right)$ and $\gamma_{s, t}\left(0, h_{t}\right)$ and the spatial and temporal variogram models $\gamma_{s}\left(\mathbf{h}_{s}\right)$ and $\gamma_{t}\left(h_{t}\right)$, respectively. Note that

$$
C_{s t}(0,0)=k_{1} C_{s}(0) C_{t}(0)+k_{2} C_{s}(0)+k_{3} C_{t}(0)
$$

Then $k_{1}, k_{2}$ and $k_{3}$ can be solved for in terms of the sill values $C_{s t}(0,0), C_{s}(0), C_{t}(0), k_{s}$ and $k_{t}$ :

$$
\begin{aligned}
& k_{1}=\frac{k_{s} C_{s}(0)+k_{t} C_{t}(0)-C(0,0)}{C_{s}(0) C_{t}(0)} \\
& k_{2}=\frac{C_{s t}(0,0)-k_{t} C_{t}(0)}{C_{s}(0)} \\
& k_{3}=\frac{C_{s t}(0,0)-k_{s} C_{s}(0)}{C_{t}(0)}
\end{aligned}
$$

Thus the allowable values for $k_{s}$ and $k_{t}$ that will ensure $k_{1}>0, k_{2} \geq 0$ and $k_{3} \geq 0$ are related to the sills of the spatial and temporal components.

\section{SOME GENERAL RESULTS}

The product-sum model exhibits several interesting and perhaps unexpected features pertaining to the sill values of the component variograms. Recall the relationships in Equations (13), (14) and (15) between a covariance and the corresponding variogram.

\subsection{Necessary and sufficient conditions}

Consider a space-time variogram of the form

$$
\gamma_{s t}\left(\mathbf{h}_{s}, h_{t}\right)=\gamma_{s t}\left(\mathbf{h}_{s}, 0\right)+\gamma_{s t}\left(0, h_{t}\right)-k \gamma_{s t}\left(\mathbf{h}_{s}, 0\right) \gamma_{s t}\left(0, h_{t}\right)
$$

Then

$$
0<k \leq \frac{1}{\max \left[\operatorname{sill} \gamma_{s t}\left(\mathbf{h}_{s}, 0\right), \operatorname{sill} \gamma_{s t}\left(0, h_{t}\right)\right]}
$$

is a necessary and sufficient condition for $\gamma_{s t}\left(\mathbf{h}_{s}, h_{t}\right)$ to be a valid space-time variogram. The proof is found in De Iaco et al. (2000a).

\subsection{Marginal variograms}

Consider a plot of the space-time variogram and view the domain as though it were two-dimensional. Then the functions $\gamma_{s t}\left(\mathbf{h}_{s}, 0\right), \gamma_{s t}\left(0, h_{t}\right)$ might be thought of as 'marginals', analogous to the idea of a marginal probability distribution for a joint probability distribution. One marginal is the trace of the space-time variogram in the 'plane' $h_{t}=0$; the second is the trace of the variogram in the 'plane' $\mathbf{h}_{s}=0$. In the constructions above, unlike the case of probability distributions, the 'marginals' are 
nearly sufficient to determine the 'joint' variogram. However, there are two additional marginals that may be of interest, particularly when examining the joint sample space-time variogram. These additional marginals are $\gamma_{s t}\left(\mathbf{h}_{s}, \infty\right)$ and $\gamma_{s t}\left(\infty, h_{t}\right)$. The sample variograms corresponding to these will indicate whether it will be necessary to incorporate a non-bounded component in the space-time variogram. This extension will be discussed later.

\section{GENERALIZED PRODUCT MODEL}

It is well known that positive linear combinations of valid models are again valid. This is equally applicable to space-time models as to spatial models. This construction and property can be extended to an integral form using a probability density function (Matern, 1960; Myers, 1988). That is, let $f(u)$ be a probability density function defined on a subinterval of the real line and $\gamma(\mathbf{h} ; u)$ be a valid variogram for each choice of $u$ in the domain of $f$. Then

$$
\gamma_{\text {new }}(\mathbf{h})=\int_{R} \gamma(\mathbf{h} ; u) f(u) \mathrm{d} u
$$

is again a valid variogram model. This construction will extend easily to vector values for $u$ by the use of joint densities and will extend to the use of probability distribution functions, in lieu of a density, by the use of a Stieltges integral. Models constructed using this general form of a positive linear combination may have quite different characteristics from phase of their constituent parts. For example, the 'hole-effect' model can be generated in this way:

$$
\gamma(r)=\int_{0}^{1}[1-\cos (r \alpha)] \mathrm{d} \alpha=1-\frac{\sin (r)}{r} \quad \text { if } r>0 \text { and } \gamma(0)=0 .
$$

Note that even though $1-\cos r$ is only semi-definite the integrated form is definite. This contrast appears again in the next section.

\section{THE CRESSIE-HUANG CONSTRUCTION}

Cressie and Huang (1999) have used a variation of this idea applied to the product model. Assume that $C\left(\mathbf{h}_{s}, h_{t}\right)$ is integrable; then it can be written in the form

$$
C\left(\mathbf{h}_{s}, h_{t}\right)=\int_{R^{k}} e^{i \mathbf{h}_{s}^{\prime} \omega} \rho\left(\omega ; h_{t}\right) k(\omega) \mathrm{d} \omega
$$

where $\rho(\omega, \cdot)$ is a continuous autocorrelation function for each $\omega$ in $R^{k}$,

$$
\begin{aligned}
& \int_{\Re_{+}} \rho\left(\omega ; h_{t}\right) \mathrm{d} h_{t}<\infty, \\
& k(\omega)>0 \text { and } \int_{\Re^{n}} k(\omega) \mathrm{d} \omega<\infty .
\end{aligned}
$$


If this construction is viewed as an integral of the product of spatial and temporal covariances, the spatial covariance is only semi-definite, although the space-time covariance is positive definite by construction.

\section{THE INTEGRATED PRODUCT MODEL}

To ensure that the resulting integral form is valid, we consider only positive definite models. The following theorem is taken from De Iaco et al. (2000a).

\section{Theorem 1}

Let $\mu(a)$ be a positive measure over $U \subseteq \Re$, and let $C_{s}\left(\mathbf{h}_{s} ; a\right)$ and $C_{t}\left(h_{t} ; a\right)$ be covariances, respectively, in $D \subset R^{k}$ and $T \subset R_{+}$, for each $a \in V \subseteq U$. Assume that the product $C_{s}\left(\mathbf{h}_{s} ; a\right) \cdot C_{t}\left(h_{t} ; a\right)$ is integrable with respect to the measure $\mu$ over $V$ for each $\mathbf{h}_{s}$ and $h_{t}$. Then for any $K>0$

$$
C_{s t}\left(\mathbf{h}_{s}, h_{t}\right)=\int_{V} K C_{s}\left(\mathbf{h}_{s} ; a\right) C_{t}\left(h_{t} ; a\right) \mathrm{d} \mu(a)
$$

is a covariance in $R^{k} \times T$.

This result can be re-written in terms of variograms:

$$
\gamma_{s t}\left(\mathbf{h}_{s}, h_{t}\right)=\int_{V} K\left[C_{t}(0 ; a) \gamma_{s}\left(\mathbf{h}_{s} ; a\right)+C_{s}(0 ; a) \gamma_{t}\left(h_{t} ; a\right)-\gamma_{s}\left(\mathbf{h}_{s} ; a\right) \gamma_{t}\left(h_{t} ; a\right)\right] \mathrm{d} \mu(a) .
$$

\section{Example 1}

Let

$$
C_{s}\left(\mathbf{h}_{s}\right)=\exp \left[-a\left|\mathbf{h}_{s}\right|^{2}\right]
$$

and

$$
C_{t}\left(h_{t}\right)=\exp \left[-a\left(h_{t}\right)^{2}\right]
$$

and

$$
f(a)=\exp (-a)
$$

the probability density function for the measure $\mu(a)$. Then the integrated product model is

$$
C\left(\mathbf{h}_{s}, h_{t}\right)=\frac{1}{1+\left(h_{t}\right)^{2}+\left|\mathbf{h}_{s}\right|^{2}} .
$$

This model is not separable, but it is a metric model even though it was not constructed by the use of a metric. That is, if $\left|\left(\mathbf{h}_{s}, h_{t}\right)\right|^{2}$ is defined as $\left(h_{t}\right)^{2}+\left|\mathbf{h}_{s}\right|^{2}$ then the model is obtained from the standard 
isotropic model $C(r)=1 /\left(1+r^{2}\right)$ using this metric. This example can be further generalized by assuming that $C_{s}\left(\mathbf{h}_{s} ; a\right)$ and $C_{t}\left(h_{t} ; a\right)$ each depend on additional parameters. The resulting model will then depend on those parameters.

\section{Example 2}

Replace $C_{t}\left(h_{t} ; a\right)$ by

$$
C_{t}\left(h_{t} ; a\right)=\exp -a\left|h_{t}\right|^{\beta}, \quad \text { where } 0<\beta \leq 2
$$

then the model is

$$
C\left(\mathbf{h}_{s}, h_{t}\right)=\frac{1}{1+\left(h_{t}\right)^{2}+\left|\mathbf{h}_{s}\right|^{\beta}} .
$$

\section{INTEGRATED PRODUCT-SUM MODELS}

The following construction is taken from De Iaco et al. (2000b).

\section{Theorem 2}

Let $\mu(a)$ be a positive measure over $U \subseteq \Re$, let $C_{s}\left(\mathbf{h}_{s} ; a\right)$ and $C_{t}\left(h_{t} ; a\right)$ be covariances, respectively, in $D \subset R^{k}$ and $T \subset R_{+}$, for each $a \in V \subseteq U$. Suppose that $C_{s}\left(\mathbf{h}_{s} ; a\right) \cdot C_{t}\left(h_{t} ; a\right)$ is integrable with respect to the measure $\mu$ over $V$ for each $\mathbf{h}_{s}$ and $h_{t}$. For any $k_{1}>0, k_{2} \geq 0$ and $k_{3} \geq 0$, then

$$
C_{s t}\left(\mathbf{h}_{s}, h_{t}\right)=\int_{V}\left[k_{1} C_{s}\left(\mathbf{h}_{s} ; a\right) C_{t}\left(h_{t} ; a\right)+k_{2} C_{s}\left(\mathbf{h}_{s} ; a\right)+k_{3} C_{t}\left(h_{t} ; a\right)\right] \mathrm{d} \mu(a)
$$

is a covariance.

\section{Case Study 3}

The methods described above have been applied to hourly average concentrations of $\mathrm{NO}_{2}$ measured during August 1997 in 18 survey stations in Milan District. After removing the seasonal component by the standard technique of moving averages (Brockwell and Davis, 1987), residuals, available for all stations, were used for the structural analysis.

The steps for generating the space-time variogram model are listed below. Examining the shapes of the sample spatial and temporal variograms, $\widehat{\gamma}_{s, t}(\cdot, 0)$ and $\widehat{\gamma}_{s, t}(\mathbf{0}, \cdot)$, exponential models were fitted for $\gamma_{s}(\cdot, a)$ and $\gamma_{t}(\cdot, a)$ whose analytical expressions are, respectively:

$$
\begin{aligned}
& \gamma_{s}\left(\mathbf{h}_{s}, a\right)=1-\exp \left\{-a \frac{|| \mathbf{h}_{s} \mid}{4414}\right\} \\
& \gamma_{t}\left(h_{t}, a\right)=1-\exp \left\{-a \frac{h_{t}}{8.22}\right\}
\end{aligned}
$$


and the sill values are 400 and 250 , respectively, for the spatial and temporal structures, i.e. $k_{2}+k_{1}=$ 400 , and $k_{3}+k_{1}=250$.

$$
\begin{aligned}
\phi(a) & =9.84 a^{2} \exp \{-2.7 a\} \\
\gamma_{s, t}\left(\mathbf{h}_{s}, 0\right) & =\int_{0}^{\infty}\left[400\left(1-\exp \left\{-a \frac{\left\|\mathbf{h}_{s}\right\|}{4414}\right\}\right)\right]\left[9.84 a^{2} \exp \{-2.7 a\}\right] \mathrm{d} a \\
& =400\left(1-\frac{2.7^{3}}{\left(2.7+\frac{\left\|\mathbf{h}_{s}\right\|}{4414}\right)^{3}}\right) \\
\gamma_{s, t}\left(\mathbf{0}, h_{t}\right) & =\int_{0}^{\infty}\left[250\left(1-\exp \left\{-a \frac{h_{t}}{8.22}\right\}\right)\right]\left[9.84 a^{2} \exp \{-2.7 a\}\right] \mathrm{d} a \\
& =250\left(1-\frac{2.7^{3}}{\left(2.7+\frac{h_{t}}{8.22}\right)^{3}}\right)
\end{aligned}
$$

The details for choosing $f(a)$ are found in De Iaco et al. (2000b, 2000c).

\section{FURTHER EXTENSIONS}

The product, product-sum and integrated product may be viewed as special cases of the integrated product-sum models. One disadvantage of these constructions is that they are all based on variograms that correspond to covariances. That is, the resulting space-time models will be bounded and have sills. While the sum of a space variogram and a temporal variogram is generally not a valid space-time variogram, even if both components correspond to covariances, adding a space variogram and/or a temporal variogram to a valid space-time variogram will result in a valid model. Let $K_{1}>0, K_{2} \geq 0$ and $K_{3} \geq 0$ be arbitrary real numbers. Then a more general model might be of the form

$$
\gamma_{G}\left(\mathbf{h}_{s}, h_{t}\right)=K_{1} \gamma_{s t}\left(\mathbf{h}_{s}, h_{t}\right)+K_{2} \gamma_{s 1}\left(\mathbf{h}_{s}\right)+K_{3} \gamma_{t 1}\left(h_{t}\right)
$$

where $\gamma_{s t}\left(\mathbf{h}_{s}, h_{t}\right)$ is a valid space-time variogram, e.g. obtained as an integrated product-sum model, $\gamma_{s 1}\left(\mathbf{h}_{s}\right)$ is a valid space variogram (not necessarily corresponding to a covariance) and $\gamma_{t 1}\left(h_{t}\right)$ is a valid time variogram (not necessarily corresponding to a covariance). Consider now the marginal variograms:

$$
\begin{aligned}
\gamma_{G}\left(\mathbf{h}_{s}, 0\right) & =K_{1} \gamma_{s t}\left(\mathbf{h}_{s}, 0\right)+K_{2} \gamma_{s 1}\left(\mathbf{h}_{s}\right) \\
\gamma_{G}\left(0, h_{t}\right) & =K_{1} \gamma_{s t}\left(0, h_{t}\right)+K_{3} \gamma_{t 1}\left(h_{t}\right) \\
\gamma_{G}\left(\mathbf{h}_{s}, \infty\right) & =K_{1} \gamma_{s t}\left(\mathbf{h}_{s}, \infty\right)+K_{2} \gamma_{s 1}\left(\mathbf{h}_{s}\right)+K_{3} \gamma_{t 1}(\infty) \\
\gamma_{G}\left(\infty, h_{t}\right) & =K_{1} \gamma_{s t}\left(\infty, h_{t}\right)+K_{2} \gamma_{s 1}(\infty)+K_{3} \gamma_{t 1}\left(h_{t}\right)
\end{aligned}
$$

It is the last two marginals, or rather the sample versions of them, that indicate whether one or more of the two components $\gamma_{s 1}\left(\mathbf{h}_{s}\right), \gamma_{t 1}\left(h_{t}\right)$ are needed and whether unbounded models must be incorporated. Cressie and Huang (1999) give such an example and used weighted least squares to determine the parameter values. 


\subsection{Fitting vs choosing models}

It is well known that there are at least two distinct stages in estimating a variogram or covariance. One pertains to the model type(s), e.g. spherical, Gaussian, etc. The second corresponds to determining the parameters of the model type(s). Of course these two steps are not completely independent. While the number of model types commonly used in geostatistics is not large-some software packages only incorporate four or five-there is still the possibility of a nested model incorporating multiple types. Once the model types are tentatively determined, an optimal fitting tool such as weighted least squares can be used to determine the parameters of the model types. While one can plot a sample omnidirectional space-time sample variogram and compare with various possible models, it is an advantage to be able to make at least an initial fit by separating the dependence on space and time. The marginal sample variograms are easier to identify and model.

\subsection{Anisotropies}

By separating the dependence on space and time in the variogram or covariance one is incorporating a non-geometric anisotropy on space-time. However, the above constructions allow two extensions that are useful. One is that the usual form of geometric anisotropy can be incorporated into the dependence on the space variables. For simplicity of presentation, the above construction focused on models that were isotropic in space, though this is not essential. Moreover, by the use of the marginal models one can model the spatial geometric anisotropy completely separately from combining the spatial marginal with the temporal model. While the construction was motivated by the need to recognize the different character of time, as compared to a Euclidean dimension, the same methodology can be used to construct zonal anisotropies in Euclidean space. In fact it is not necessary to split an $n$-dimensional space into an $(n-1)$-dimensional and a one-dimensional space, the methodology will work equally well for splitting into different dimensional combinations. In that case one can then incorporate geometric anisotropies into each lower dimensional space. This is discussed in Myers et al. (2000).

\section{MODELING THE PLUME FUNCTION}

We turn now to the use of the dual kriging estimator and its extension to space-time form. Recall that the (ordinary) kriging estimator is usually written in the form

$$
Z^{*}\left(\mathbf{s}_{0}, t_{0}\right)=\sum_{i=1}^{n} \lambda_{i} Z\left(\mathbf{s}_{i}, t_{i}\right)
$$

where the coefficients $\lambda_{i} ; i \ldots n$ are obtained from a system of linear equations and are functions of $\mathbf{s}_{0}, \mathbf{t}_{0}$. The form of the estimator given in Equation (59) is essentially dimension free. Under the assumption of a constant mean the equations for determining the coefficients in Equation (59),

$$
\sum_{i=1}^{n} \lambda_{i} \gamma_{s t}\left(\mathbf{s}_{i}-\mathbf{s}_{j}, t_{i}-t_{j}\right)+\mu=\gamma_{s t}\left(\mathbf{s}_{0}-\mathbf{s}_{j}, t_{0}-t_{j}\right) ; j=1, \ldots, n \text { and } \sum_{i=1}^{n} \lambda_{i}=1
$$


are also dimension free. The dual form of Equation (59) is

$$
Z^{*}\left(\mathbf{s}_{0}, t_{0}\right)=\sum_{i=1}^{n} B_{i} \gamma_{s t}\left(\mathbf{s}_{0}-\mathbf{s}_{i}, t_{0}-t_{i}\right)+A,
$$

where

$$
\sum_{i=1}^{n} B_{i} \gamma_{s t}\left(\mathbf{s}_{i}-\mathbf{s}_{j}, t_{i}-t_{j}\right)+A=Z\left(\mathbf{s}_{j}, t_{j}\right) ; j=1, \ldots, n \text { and } \sum_{i=1}^{n} B_{i}=0
$$

\subsection{Space-time non-stationarities}

In the purely spatial context the ordinary kriging estimator can easily be extended to the universal kriging estimator; in fact there is no change in the form of the estimator, the kriging equations change and the form of those equations depends on an assumed functional form for the non-constant mean. In the spatial context the mean is usually assumed to be modeled as a polynomial in the spatial coordinates, i.e. a linear combination of monomials in the spatial coordinates. These monomials have several important properties. First of all, they are linearly independent. Secondly, they must satisfy the unbiasedness conditions; in the dual form of the universal kriging estimator they must satisfy what is called in the radial basis function literature, polynomial solvency conditions.

However in practice the universal kriging estimator is not often used in geostatistical applications. One tool for avoiding it is the use of a moving search neighborhood. Other authors use various techniques to 'remove' the non-stationarity, such as fitting a trend surface to the data and modeling the variogram using the residuals and applying ordinary kriging to the residuals. The fitted trend surface is then added back to the kriged values. Median polish has also been used to 'remove' the nonstationarity, because of well-known problems with modeling the variogram using residuals from trend surfaces. These alternative approaches to dealing with non-stationarities in space can be extended to space-time.

\subsection{The dual form of the universal kriging estimator in space-time}

Although the form of the ordinary kriging estimator and the universal kriging estimator are the same, in dual form they are not the same. The basis functions for the non-constant mean function will appear in the dual form of the estimator. Moreover there is no simple way to use a moving search neighborhood in Equation (62). It is therefore important to consider possible functional forms for a non-constant mean for a function defined on $R^{k} \times T$. Although polynomials in the spatial coordinates are rather natural, for the temporal dependence a Fourier series is more plausible. $\mathrm{E}[Z(\mathbf{s}, t)]$ might then be a sum of a spatial mean component, a temporal mean component and perhaps a product of the two. Dimitrakopoulos and Luo (1994) have shown that the unbiasedness conditions are much more restrictive in the space-time case than in the purely spatial context.

\subsection{The general form of the approximating function}

The form of the equations is however dependent on an auxiliary assumption, i.e. $\mathrm{E}[Z(\mathbf{s}, t)]$ is assumed to be a function of $t$ and/or the coordinates of $\mathbf{s}$. For a purely spatial case the mean is usually written as 
a polynomial in the coordinates of $\mathbf{s}$. While this can be extended space-time by using a polynomial in $\mathbf{s}$ and $t$, it is perhaps more natural to include sin, cos terms (as functions of time) as well. When the trigonometric terms are included the auxiliary equations are different from those for purely polynomial functions, this has been discussed in Dimitrakopoulos and Luo (1994). For now the representation of the mean will be given in slightly simpler form,

$$
\mathrm{E}[Z(\mathbf{s}, t)]=\sum_{j=0}^{p} f_{j}(\mathbf{s}, t)
$$

where the $f_{j}(\mathbf{s}, t) \mathrm{s}$ are assumed to be linearly independent in space-time. Let the point $\left(\mathbf{s}_{i}, t_{i}\right)$ be abbreviated as simply $X_{i}$. Then in vector form the kriging estimator becomes

$$
Z^{*}\left(\mathbf{s}_{0}, t_{0}\right)=\mathbf{Z} \mathbf{\Lambda}
$$

where

$$
\mathbf{Z}=\left[Z\left(X_{1}\right), \ldots, Z\left(X_{n}\right), 0, \ldots, 0\right]
$$

and

$$
\boldsymbol{\Lambda}=\left[\lambda_{1}, \ldots, \lambda_{n}, \mu_{0}, \ldots, \mu_{p}\right]^{T}
$$

$\Lambda$ is of course the solution vector for the kriging equations. By simple linear algebra it is easily seen that the Equation (61) can be re-written in the form (Matheron, 1973; Myers, 1988, 1992):

$$
Z^{*}\left(\mathbf{s}_{0}, \mathbf{t}_{0}\right)=\sum_{i=1}^{n} b_{i} \gamma\left(\mathbf{s}_{0}-\mathbf{s}_{i}, \mathbf{t}_{0}-\mathbf{t}_{i}\right)+\sum_{j=0}^{p} a_{j} f_{j}\left(\mathbf{s}_{0}, \mathbf{t}_{0}\right)
$$

The coefficients $b_{i}, i=1, \ldots, n$ and $a_{j}, j=0, \ldots, p$ are also obtained from a linear system of equations, the coefficient matrix is exactly the same as for the solution vector $\boldsymbol{\Lambda}$; the right hand side of the system, however, is $\mathbf{Z}^{\mathrm{T}}$. In this form and in the numerical analysis literature Equation (65) is known as the radial basis function interpolator (Myers, 1992, 1994). Thus the problems associated with constructing the approximating function Equation (65) in the space-time context are entirely related to choosing and fitting valid space-time variograms (or covariances).

\section{PROPERTIES OF $Z^{*}(\mathbf{s}, t)$}

From the equations for the coefficients, it is easy to see that

$$
\sum_{i=1}^{n} b_{i}=0
$$

Hence, if the variogram has a sill, then for points in space-time sufficiently far away from all the data locations the variogram is a constant. This forces the first sum in Equation (65) to be zero. This is 
asymptotically true even if the variogram does not have a sill. Therefore, when extrapolating in time and/or space, the values of $Z^{*}$ are determined only by the second sum. Even when the variogram is unbounded (but it still must satisfy a growth condition) this property is asymptotically true. Moreover the second sum is an unbiased estimator of the mean of $Z$.

\subsection{Integrability}

Some characteristics of the plume can be determined by integrating $Z(\mathbf{s}, t)$, which in turn is approximated by integrating $Z^{*}(\mathbf{s}, t)$. It is well known in the geostatistics literature that one can use 'block' kriging to estimate the average value over a region. This requires computing average values of the variogram (or covariance), so-called 'point-to-block' values. Normally this is done numerically in the software. What may not be so well known is that the integral of $Z^{*}(\mathbf{s}, t)$ is the same as the 'block' kriging estimator. Note that, in a space-time context, there are at least three forms of the integral that may be of interest: (i) the integral over a region in space-time; (ii) the integral over a region in space (which will then be a function of time); (iii) the integral over an interval in time (which will then be a function defined in space). These may be in cumulative form or in average form. Likewise 'data' may be non-point in space-time, only in space or only in time. This means that one may have to consider regularization in these three forms. A note of caution: averaging over space and then averaging over time may not be the same as averaging in space-time. Note that integrability of $Z^{*}(\mathbf{s}, t)$ only requires the continuity of the the variogram and the mean functions.

\subsection{Differentiability}

Rates of change are naturally expressed as derivatives, most often with respect to time. Again it can be shown that a (partial) derivative of $Z^{*}(\mathbf{s}, t)$ is the estimator of the mean square derivative of $Z(\mathbf{s}, t)$. It may also be of interest to mix integrability and differentiability - for example, a time derivative of an integral over space. Although integrability is a weak condition, the same is not true for differentiability. Some variograms are differentiable at the origin and others are not. A Gaussian model is infinitely differentiable at the origin but most of the other spatial models in common use are not differentiable. It will also be seen that differentiability with respect to a spatial coordinate is not the same as differentiability with respect to time. Finally note that the space-time variogram constructions given in the preceding sections 'separate' the differentiability with respect to time and space, thus the estimated plume function may be time differentiable but not differentiable with respect to spatial coordinates.

\section{COMPUTATIONAL ASPECTS AND CONCERNS}

The usual geostatistical software does not incorporate either the space-time variogram estimation algorithms described above nor the kriging, in its usual format, with the new space-time models. De Cesare et al. (2000c) have addressed these problems by modifying the kriging and variogram programs from the GSLIB package. A program for simultaneous fitting of the time series, to deal with temporal non-stationarities, is also found in De Cesare et al. (2000c). More recently the same authors have developed MATLAB codes for the dual form of space-time kriging.

For kriging in its usual form for spatial problems, it is common practice to use a moving search neighborhood, thus limiting the size of the coefficient matrix and avoiding numerical problems in 
solving the linear system. The possibilities for introducing discontinuities as a result of using the moving search neighborhood are largely hidden in this form. However, the use of a moving search neighborhood with the dual form of kriging means that it would be necessary to construct a global function by piecing together locally fitted functions. The alternative of using a unique neighborhood may not be completely satisfactory when applied to space-time problems since the total number of data locations (in space-time) may be very large. Faul and Powell (1999) have developed an iterative algorithm for determining the coefficients in a radial basis function interpolator. Since the radial basis function interpolator is the same as the dual form of the kriging estimator, their solution can be applied to the dual form of the space-time kriging. Hence if the number of data locations is too large the equation solver can be modified to incorporate the Faul and Powell algorithm.

\section{SOME CONCLUDING REMARKS}

\subsection{Product models}

While it was seen that the product model in space-time has some disadvantages, a spatial product exponential model is often used in hydrology. This is of the form

$$
C\left(h_{x}, h_{y}, h_{z}\right)=C_{0} \exp \left(-\left(\left|h_{x}\right| / a_{x}\right)-\left(\left|h_{y}\right| / a_{y}\right)-\left(\left|h_{z}\right| / a_{z}\right)\right) \text {. }
$$

$h_{x}, h_{y}, h_{z}$ are the increments in the three directions (3-dimensional Euclidean space). $a_{x}, a_{y}, a_{z}$ are usually referred to as the 'correlation lengths'; these are proportional to the respective effective ranges of dependence. This model is of course a zonal anisotropy, although that characteristic is often not mentioned in the literature. $C_{0}$ is the 'sill', and in variogram form the model is

$$
\gamma\left(h_{x}, h_{y}, h_{z}\right)=C_{0}\left[1-\exp \left(-\left(\left|h_{x}\right| / a_{x}\right)-\left(\left|h_{y}\right| / a_{y}\right)-\left(\left|h_{z}\right| / a_{z}\right)\right)\right] .
$$

\subsection{The nugget effect and space-time variograms}

For a variogram $\gamma(\mathbf{h})$ defined on space, the nugget is a discontinuity at the origin. That is, it is

$$
\operatorname{Lim}_{|\mathbf{h}| \rightarrow 0} \gamma(\mathbf{h}) .
$$

Of course, by definition, $\gamma(0)=0$. However, this limit may not have a unique value if the variogram is allowed to incorporate a zonal anisotropy, i.e. the value of the limit may depend on the path as $|\mathbf{h}| \rightarrow 0$. In general a space-time variogram will be analogous to a zonal anisotropy.

The nugget effect is usually interpreted in one of two ways: (a) as a variance it incorporates the variance of measurement error; (b) it appears because of short range variability, i.e. with a range of dependence that is less than the shortest inter-data distance. Incorporating the nugget effect into the variogram model is a 'conservative' decision; it decreases the amount of spatial dependence that is modeled. This second interpretation is particularly relevant in the case of the marginal space and marginal time variograms. As shown above these are naturally estimated as time and space (respectively) averages of the space and time sample variograms. That is, the marginal space variogram is estimated by averaging, over all data time points, the space sample variograms and 
analogously for the marginal time variogram. Hence the marginal space variogram incorporates short (time) variability and the marginal time variogram incorporates short (spatial distance) variability. These are still not quite the magnitudes of the discontinuities, however. The nugget effects, if any, appearing in the models for the respective marginal variograms will be given by

1.

$$
\operatorname{Lim}_{\mathbf{h}_{s} \rightarrow 0} \gamma_{s t}\left(\mathbf{h}_{s}, 0\right)
$$

and

2.

$$
\operatorname{Lim}_{h_{t} \rightarrow 0} \gamma_{s t}\left(0, h_{t}\right)
$$

Note that in general

$$
\operatorname{Lim}_{\mathbf{h}_{s} \rightarrow 0} \gamma_{s t}\left(\mathbf{h}_{s}, h_{t}\right) \neq \gamma_{s t}\left(0, h_{t}\right)
$$

and

$$
\operatorname{Lim}_{h_{t} \rightarrow 0} \gamma_{s t}\left(\mathbf{h}_{s}, h_{t}\right) \neq \gamma_{s t}\left(\mathbf{h}_{s}, 0\right)
$$

Thus there are several different possible concepts for 'nugget effect' for a space-time variogram. Several of the above are special cases of

$$
\operatorname{Lim}_{\mathbf{h}_{s} \rightarrow 0, h_{t} \rightarrow 0} \gamma_{s t}\left(\mathbf{h}_{s}, h_{t}\right)
$$

which will almost certainly depend on the path as $\left(\mathbf{h}_{s}, h_{t}\right) \rightarrow(0,0)$.

\section{SUMMARY}

The dual kriging form of the kriging estimator can be used to model contaminant plumes by using general space-time variogram models such as the product-sum or integrated product sum. These variograms can be fitted to space-time data by using the space and time marginal variograms. By fitting an analytic form of the function representing the plume concentrations in space-time, other plume characteristics can be estimated.

\section{REFERENCES}

Brockwell PJ, Davis RA. 1987. Time Series: Theory and Methods. Springer-Verlag: New York.

Cressie N, Huang H. 1999. Classes of nonseparable, spatio-temporal stationary covariance functions. JASA 94: 1330-1340.

De Cesare L, Myers D, Posa D. 1997. Spatial-temporal modeling of $\mathrm{SO}_{2}$ in Milan District. In Geostatistics Wollongong'96, Vol. 2, Baafi EY, Schofield NA (eds). Kluwer Academic Publishers; 1031-1042.

De Cesare L, Myers D, Posa D. 2000a. Product-sum covariance for space-time modeling. Environmetrics 12: 11-23.

De Cesare L, Myers D, Posa D. 2000b. Estimating and modeling space-time correlation structures. Statistics and Probability Letters 51(1): 9-14.

De Cesare L, Myers D, Posa D. 2002. FORTRAN programs for space-time modeling. Computers \& Geosciences 28: $205-212$. 
De Iaco S, Myers D, Posa D. 2000a. Space-time analysis using a general product-sum model. Statistics and Probability Letters 52(1): 21-28.

De Iaco S, Myers D, Posa D. 2002. Nonseparable space-time covariance models: some parametric families. Mathematical Geology 34: 23-42.

De Iaco S, Myers D, Posa D. 2000c. Total air pollution and space-time modeling. In geoENV III, Geostatistics for Environmental Applications, Monestiez P, Allard D, Froidevaux R (eds). Kluwer Academic Publishers; 45-56.

Dimitrakopoulos R, Luo X. 1994. Spatiotemporal modeling: covariances and ordinary kriging systems. Geostatistics for the Next Century. Kluwer Academic Publishers; 88-93.

Faul AC, Powell MJD. 1999. Krylov subspace methods for radial basis function interpolation. Numerical Analysis. Chapman \& Hall/CRC Res. Notes Math 420: 115-141.

Kyriakidis PC, Journel AG. 1999. Geostatistical space-time models: a review. Mathematical Geology 31: 651-684.

Madych WR, Nelson SA. 1988. Multivariate interpolation and conditionally positive definite functions. Approx. Theory \& its Appl. 4: 77-89.

Matern B. 1960. Spatial Variation. Medd. Stetens Skogsforskniginst Swed. 5.

Matheron G. 1973. The Intrinsic Random Functions and Their Applications. Adv. in Appl. Prob. 5: 439-468.

Myers DE. 1988. Interpolation with positive definite functions. Sciences de la Terre 28: 251-265.

Myers DE. 1991. On variogram estimation. In Proceedings of the First Inter. Conf. Stat. Comp., Cesme, Turkey, 30 March2 April 1987, Vol. II. American Sciences Press; 261-281.

Myers DE. 1992. Cokriging, radial basis functions and the role of positive definiteness. Computers Math. Applications 24: 139148.

Myers DE. 1994. Statistical methods for interpolation of spatial data. J. Applied Science and Computations 1: 283-318.

Myers DE, Journel AG. 1990. Variograms with zonal anisotropies and non-invertible kriging systems. Mathematical Geology 22: 779-785.

Myers DE, De Iaco S, Posa D, De Cesare L. 2002. Space-time radial basis functions. Computers and Math. Applications 34: 539-549.

Posa D. 1993. A simple description of spatial-temporal processes. Computational Statistics \& Data Analysis 15: 425-438.

Rodriguez-Iturbe I, Meija JM. 1974. The design of rainfall networks in time and space. Water Resources Research 10: 713-728.

Rouhani S, Hall TJ. 1989. Space-time kriging of groundwater data. In Geostatistics, Vol. 2, Armstrong M (ed.), Kluwer Academic Publishers; 639-651.

Rouhani S, Myers D. 1990. Problems in space-time kriging of geohydrological data. Mathematical Geology 22: 611-623. 